Auf den letzten Seiten seiner Aufzeichnungen versucht sich Wilerzol an einer Sinnstiftung für die Schrecken seiner Soldatenjahre, die "Strapazen" und "blutigen Opfer" ${ }^{11}$ : „Es ist vielleicht eine notwendige Periode gewesen, bzw. ist es noch - ein Gährungsprozeß, den wir alle, den das deutsche Volk, durchmachen müßen. "12 Dass es mit dem deutschen Volk wieder aufwärts gehen wird, dessen ist er sich gewiss. Diese Hoffnungen auf eine positive Zukunft erfüllten sich für Deutschland bekanntermaßen nicht. Vielmehr steuerte alles auf ein noch größeres Unglück zu. Was die folgenden Jahre für Otto Wilerzol bereithielten, ist nicht bekannt.

Tobias Thelen

\title{
Eine Musikaliensammlung von Königin Charlotte von Württemberg
}

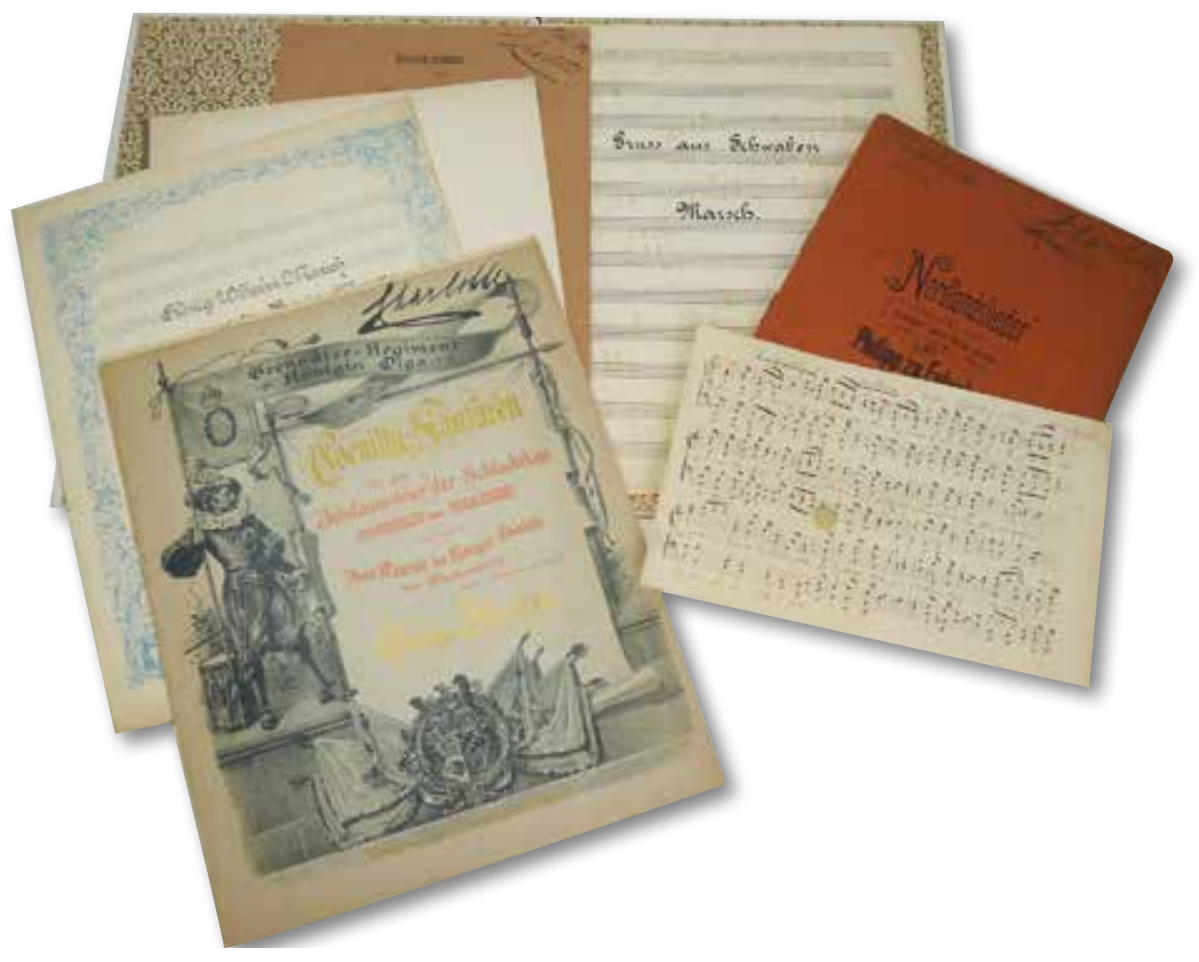

Im Dezember 2019 erhielt die Württembergische Landesbibliothek eine kleine Notensammlung aus dem Landesmuseum Württemberg, die Königin Charlotte von Württemberg (1864-1946) zugeordnet werden kann. Da im Landesmuseum keine Noten inventarisiert werden und die Titel aufgrund ihrer Provenienz hervorragend in die Musiksammlung der Landesbibliothek passen, bot sich die Übergabe an.

Charlotte, die letzte Königin von Württemberg und eine Frau mit vielseitigen Interessen und Begabungen, hinterließ im Lande viele Spuren: im sozialkaritativen Bereich, als überzeugte Vertreterin der
Frauenemanzipation und im Bereich der Schönen Künste. Das kulturelle Engagement von Wilhelm II. war zweifelsfrei groß und "sein Programm, Stuttgart zu einem Kulturzentrum zu machen, hat sich durchaus erfüllt" (Janzen, 247). Charlotte jedoch stand inm hier in nichts nach, kann vielleicht sogar als "die Interessiertere von beiden" angesehen werden (Janzen, 45). Sie besuchte regelmäßig mit ihrem Mann und bestens vorbereitet Opern- und Theateraufführungen in Stuttgart (Janzen, 50-51), reiste auch ohne seine Begleitung zu den Bayreuther Festspielen (Haug, 86). Darüber hinaus musizierte sie selbst, häufig in Bebenhausen mit ihren Schwestern. Neben einem Hammerflügel aus 
Stuttgarter Produktion (Richard Lipp) standen dort auch andere Instrumente zur Verfügung (Haug, 52).

Die vorliegende Notensammlung kann aufgrund verschiedener Merkmale Charlotte zugeordnet werden. Deshalb liegt es nahe, Fragen an diese Sammlung zu stellen, die aus dem Wissen über Charlottes Person resultieren. So etwa könnte die Anzahl der weiblichen Komponisten ermittelt und mit Charlottes Einsatz für die Stellung und die Rechte der Frau in Verbindung gebracht werden. Auch wenn im Folgenden solche Fragen aufgeworfen werden, sind mögliche Schlussfolgerungen in den meisten Fällen direkt wieder zu relativieren. Dies hat verschiedene Gründe, allen voran ist hier der geringe Umfang dieser Sammlung zu nennen. Es kann nicht davon ausgegangen werden, dass eine lediglich 34 Titel umfassende Sammlung das musikalische Interesse Charlottes und ihre Betätigung in diesem Bereich vollständig widerspiegelt. Weshalb sollte die Königin Notenausgaben in breiter Auswahl in ihrem Privatbesitz gehabt haben, wenn ihr die ganze Hofbibliothek zur Verfügung stand? Diese nutzte sie nachgewiesenermaßen sehr häufig (Janzen, 101), und ein Teil davon war seit 1750 auch das nicht mehr benötigte Notenmaterial des Hoftheaters. Weshalb sollte sich, um nur ein weiteres Beispiel derartiger Fragen zu nennen, der Kontakt zu Musikerinnen und Komponistinnen in physischem Notenmaterial niederschlagen, wenn Charlotte doch allein schon durch ihre alltäglichen Aufgaben so gut vernetzt war, dass sie regelmäßig direkten Austausch über Musik führen konnte? So etwa mit einzelnen Mitgliedern des auch von Königin Charlotte protegierten Schwäbischen Frauenvereins, dem z.B. auch die Pianistin Johanna Klinckerfuß angehörte, eine Schülerin von Sigmund Lebert und Franz Liszt, die mit dem Stuttgarter Klavierbauer und -restaurator Apollo Klinckerfuß verheiratet war, im gemeinsamen Salon Kontakt zu Johannes Brahms, Edward Grieg, Hans von Bülow und dem Dirigenten Fritz Busch hatte - um nur einige zu nennen - und auch mit Clara Schumann konzertierte.

Die folgende Bestandsaufnahme kann deshalb nur einen kleinen Einblick in das musikalisch-kulturelle Umfeld Charlottes geben. Schon bekanntes Wissen über die letzte Königin von Württemberg kann aus dieser Perspektive untermauert werden, jedoch kaum umgekehrt neues Wissen aus diesem kleinen Notenschatz abgeleitet werden.

Die Sammlung umfasst 34 Titel, die in gedruckten Ausgaben oder handschriftlich vorliegen, nicht immer vollständig sind und sich in ganz unterschiedlichem konservatorischem Zustand befinden. Doch selbst die Werke, die hier nur in Form von Titelblättern erhalten sind, legen Zeugnis von Charlottes Beschäftigung mit Musik ab.

21 Titel enthalten Widmungen (verschiedene Empfänger), 16 sind von Charlotte signiert.

Von den 34 Titeln können nur vier weiblichen Komponistinnen zugeordnet werden (Pauline Erdmannsdörfer-Fichtner: Türkische Liebeslieder, Fürstin Julie von Waldburg-Wurzach: Frau Nachtigall, Die wilden Nelken und An die Ferne!). Bei zwei Titeln kann aufgrund abgekürzter Vornamen und mangelnder biographischer Informationen keine Aussage getroffen werden.

Blickt man weiter auf die Urheber der in der Sammlung enthaltenen Werke, fällt auf, dass es sich durchweg um zeitgenössische Komponisten handelt. Darunter sind Komponisten und Musiker mit Landesbezug, wie Hugo Wehrle, der ab 1868 Mitglied der Württembergischen Hofkapelle Stuttgart war und mit Vier Liedern für eine mittlere Stimme mit Begleitung des Pianoforte (Op. 12) vertreten ist, oder Militärmusiker wie Hermann Bley aus dem 1. Württembergischen Ulanen-Regiment König Karl I. Nr. 19 in Ulm (Schnebel) oder der Militärkapellmeister beim 4. Württembergischen InfanterieRegiment Nr. 122 in Heilbronn Wilhelm Widmann (Schnebel). Von Bley liegt ein Jubiläums-Marsch über das Lied "Preisend mit viel schönen Reden" (Op. 2) vor, von Widmann ein König WilhelmMarsch und eine Königin Charlotte-Gavotte, beide handschriftlich und beide mit entsprechenden Widmungen versehen.

Der Mannheimer Robert Kahn, Komponist und Korrepetitor, ist mit einer Liedsammlung vertreten.

Von Rudolf Fr. Engel liegt eine handschriftliche Fassung von Sechs Gesängen für eine Singstimme mit Klavier vor, die Ihrer Majestät "Charlotte" Königin von Württemberg in tiefster Ehrfurcht gewidmet ist. Die Widmung stammt aus dem Jahr 1908, die Lieder wurden laut einzelner Vermerke jeweils nach dem Notentext in den Jahren 1907 und 1908 
in Reichenberg und Stuttgart komponiert (mit einer Ausnahme: Das Mädchen mit den roten Hündchen entstand bereits 1905 in Josefstadt). Engel wurde 1883 in Ruppersdorf bei Zittau geboren und studierte in Stuttgart Kirchenmusik. Er komponierte neben Instrumentalem auch verschiedene Lieder (Hader, Sp. 562). Die vorliegenden sechs Lieder beruhen auf Texten bekannter Dichter wie Mörike (Er ist's) und Heine (Mädchen mit den roten Hündchen), aber auch auf Texten von Verfassern mit vermutlich nur lokalem Bekanntheitsgrad - jeweils aus Charlottes neuer und aus seiner Heimat: So findet sich etwa ein Lied mit dem Text Gut' Nacht! von Irene Wahlström Cannstatt-Stuttgart und Die Träne von Theodor Hutter - Reichenberg.

Blickt man auf die Widmungsträger ist Charlotte acht Mal die Empfängerin einer Widmung, ihr Mann vier Mal, auf einer Handschrift von Louis Ritters Gruss aus Schwaben zusammen mit Charlotte (Componiert und Ihren Majestäten dem König Wilhelm II. und der Königin Charlotte von Württemberg in tiefster Ehrfurcht gewidmet). Philipp zu Eulenburg ist mit sieben Werken vertreten, eines davon besteht aus drei Teilen. Von den fünf Widmungen gelten zwei seiner Mutter, eine Sr. Kaiserlichen und Königlichen Hoheit dem Kronprinzen Wilhelm, eine seiner Frau Gräfin Augusta Sandels und eine Königin Charlotte. Hier handelt es sich um Weihnachtsgesänge, die er Charlotte als bescheidene Weihnachtsgabe im Jahr 1893 zueignete, so der handschriftliche Vermerk über dem Inhaltsverzeichnis des Drucks. Charlotte signierte auf dem Einband mit 1893 Charlotte. Darüber hinaus sind als Widmungsträger König Karl von Württemberg zu nennen (Karl Eichler: Einzug der Württemberger, Op. 8 und ferner der Militär- musiker Hermann Bley: Jubiläums-Marsch, Op. 2), Katharina von Württemberg (Julie von WaldburgWurzach: Frau Nachtigall, Großherzog Prinz Emil von Hessen (Fred Beyer: Six Morceaux Elégants pour le Piano sur des Airs Allemands favoris, Op. 96, Nr. 6), Großherzogin Marie von MecklenburgStrelitz (Charles Voss: Fantaisie de Concert pour le Piano) und Fürstin Alma von Carolath-Beuthen (Xaver Scharwenka: Impromptu für das Pianoforte, Op. 17).

Bei den handschriftlich überlieferten Noten mit Widmungen dürfte es sich wohl um Autographen handeln.

Charlotte versah 16 Notenausgaben mit ihrem Namen, manches Mal einem Jahr oder Datum und ab und an auch mit einem Ort. Diese Angaben stammen überwiegend aus den 1890er Jahren, eine liegt mit 1887 früher (Heinrich Alberti: Fantaisies Brillantes pour deux Pianos) und die jüngste von 1908 findet sich auf Robert Kahns Liederalbum. Auffällig sind in diesem Kontext die Druckausgaben von Werken Philipp zu Eulenburgs. Von den neun Bänden sind sieben mit Charlotte versehen, vier davon zusätzlich mit Berchtesgaden und alle bis auf eine (Die Tanne) weisen Jahre zwischen 1891 und 1897 auf. 1890 wurde Otto von Bismarck von Kaiser Wilhelm II. entlassen - Philipp zu Eulenburg hatte wohl seine Finger im Spiel und gewann dann zunehmend politischen Einfluss. Er war zwischen 1890 und 1894 Gesandter, u.a. in Stuttgart. Im Jahr 1891 bekam Württemberg nach dem Tod König Karls mit Wilhelm und Charlotte ein neues Königspaar. Zeigt sich zu Eulenburgs Einflussnahme auch im Verhältnis zu Charlotte? Eine Ausgabe seiner Weihnachtsgesänge erhielt Charlotte 1893 als 
Weihnachtsgeschenk. In seinen Briefen und Tagebucheinträgen dieser Zeit spricht er aber lediglich über König Wilhelm II. und das nicht gut - hier geht es um die Gefährdung der Regierung „durch das Zusammenwirken [des Fürsten] mit dem König von Württemberg" (Haller, 262). Suchte er auf anderer Ebene den Kontakt zu Charlotte und eine Einflussnahme?

Vier der Eulenburg-Notendrucke sind mit Berchtesgaden und September 1897 beschriftet. Zu Berchtesgaden können verschiedene Vermutungen angestellt werden. Charlotte war regelmäßig auf Kur in Bad Gastein und könnte in Berchtesgaden Halt gemacht haben. Diese Reisen fanden traditionell aber im Monat Mai statt (Haug, 83). Seit 1899 war Charlotte auch immer wieder auf Schloss Neubeuern

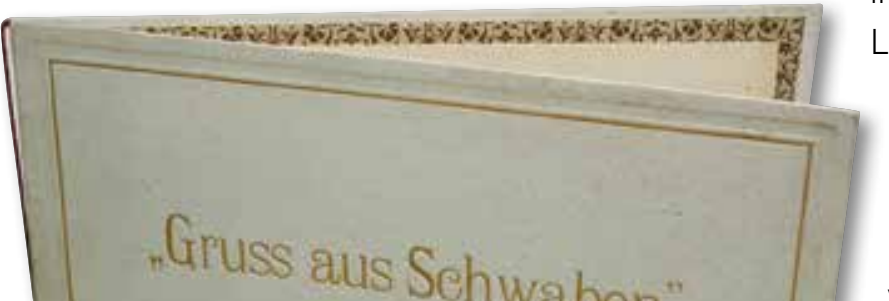
in der Württembergischen Landesbibliothek aufbewahrt wird, zeugt hingegen von der musikalisch-kulturellen Mobilität und Aufgeschlossenheit der letzten Königin von Württemberg. geordnet werden kann.

Auf einem Foto von 1934 ist Charlotte mit ihrem neuen Mercedes $500 \mathrm{~N}$ zu sehen, der ihr von da an für ihre zahlreichen Ausflüge und Reisen diente (Haug, 87). Die Notensammlung Charlottes, die nun ne Oper!) und Stücke nur für Klavier. Auch ein Blick Sammlung nur einen kleinen Teil von Charlottes musikalischer Realität widerspiegelt und ebenfalls nur in einen Bereich ihres kulturellen Umfelds einaben zu Gast (Haug, 86). Vielleicht hat sie bereits zwei Jahre zuvor Gräfin Julie von Degenfeld-

Schonburg besucht?

Bei den vielfältigen

Reisen, die Charlotte über viele Jahre hinweg unternommen hat, liegt es in jedem Fall nahe, dass sie den Berchtesgadener Aufenthalt im September 1897 mit weiteren Reisezielen verbunden hat. Und auf fast allen dieser Reisen spielte die Musik eine bedeutende Rolle: Bei den Verwandten in Nachod und in Schlesien fanden Hauskonzerte statt (darunter auch mit tschechischen Musikern, was die Ausgabe eines Werks von E. Chvála in der Sammlung Charlottes erklären könnte), man unternahm Ausflüge nach Prag und Wien. In Neubeuern bewegte sich Charlotte in einem Freundeskreis namhafter Literaten, Komponisten und Musiker und in Bayreuth besuchte sie die Festspiele (Haug, 83-87)
Ute Becker Abbildungen: Sonja Brandt

Literatur:

Hader, Widmar (Hrsg.): Lexikon zur deutschen Musik-Kultur. Böhmen, Mähren, Sudetenschlesien. München 2000. Band 1: A-L. Sp. 562.

Haller, Johannes (Hrsg.): Aus 50 Jahren. Erinnerungen, Tagebücher und Briefe aus dem Nachlaß des Fürsten Philipp zu Eulenburg-Hertefeld. Berlin 1923.

Haug, Hans: Königin Charlotte von Württemberg. Bebenhausen wurde ihr zur Heimat. Tübingen 2015.

Janzen, Brigit: König Wilhelm II. als Mäzen. Kulturförderung in Württemberg um 1900. Frankfurt am Main u.a. 1995. Diss.

Schnebel, Hanns-Helmut: Lexikon zur Militärmusik in Württemberg www.wlb-stuttgart.de/literatursuche/fachinformationen/ musik/links/landesmusikgeschichte-baden-wuerttemberg/ lexikon-zur-militaermusik-in-wuerttemberg/ [aufgerufen am 14.02.2020]

Weizsäcker, Emilie von: Geschichte der fünfundzwanzigjährigen Wirksamkeit (1875-1898) des unter dem Protektorat Ihrer Majestät der Königin Charlotte von Württemberg stehenden Schwäbischen Frauenverein zur Förderung höherer Bildung und Erwerbsfähigkeit des weiblichen Geschlechts. Stuttgart 1898. https://web.archive.org/web/20160304123531/http:// 